\title{
Chromosomal aberrations in patients with benign, sporadic pheochromocytomas
}

Benign, sporadic pheochromocytomas can be subdivided into two types that are related to the same chromosomal aberrations as are the two major groups of hereditary pheochromocytomas. This finding demonstrates that sporadic pheochromocytomas probably follow similar pathways of tumorigenesis as hereditary ones.

Pheochromocytomas are often associated with hereditary tumor syndromes, such as multiple endocrine neoplasia type 2 and von Hippel-Lindau disease, but they can also occur sporadically.

Previous studies, which used conventional comparative genomic hybridization $(\mathrm{CGH})$, showed that pheochromocytomas can be related to chromosomal aberrations in various regions, especially in $1 \mathrm{p}, 3 \mathrm{p}, 3 \mathrm{q}$, and chromosome 22. To obtain more detailed information on these aberrations, van Nederveen et al. used a new, highresolution method called array $\mathrm{CGH}$ in 36 patients with sporadic, benign pheochromocytomas.

44 ...sporadic pheochromocytomas probably follow similar pathways of tumorigenesis as hereditary ones 77

"In contrast to what we expected, we did not find small losses or gains, but confirmed the picture of losses and gains that were detected in previous studies using conventional CGH," says Francien H. van Nederveen (Erasmus MC, The Netherlands). The high-resolution analysis revealed loss of $1 \mathrm{p}$ with or without loss of $3 q$ in 20 of the 36 cases, and loss of $3 p$ with or without loss of $11 p$ in 11 cases. These patterns are comparable to those that are found in patients with pheochromocytomas related to multiple endocrine neoplasia type 2 and von
Hippel-Lindau disease, respectively. In addition, loss of $22 \mathrm{q}$ and loss of $21 \mathrm{q}$ were observed in more patients than expected, which suggests a greater involvement of these regions in the development of pheochromocytomas than previous studies did.

As a next step, the investigators would like to compare the patterns of chromosomal aberrations in patients with malignant pheochromocytomas with those in patients with benign sporadic pheochromocytomas. "Eventually, we hope to come up with a diagnostic test by which clinical behavior of pheochromocytoma can be assessed in routine clinical practice, which will then influence postoperative clinical management," explains van Nederveen.

\section{Julianna Vig}

Original article van Nederveen, F. H. et al. Array-comparative genomic hybridization in sporadic benign pheochromocytomas. Endocr. Relat. Cancer 16, 505-513 (2009). 